\title{
Misleading Serum Procalcitonin Elevation in Heatstroke
}

\author{
Marco Enzo Tau, Melissa Cocca \\ Ospedale Regionale di Lugano, Lugano, Switzerland
}

Received: 08/07/2017

Accepted: 20/07/2017

Published: $26 / 07 / 2017$

How to cite this article: Tau ME, Cocca M. Misleading serum procalcitonin elevation in heatstroke. EJCRIM 2017;4: doi:10.12890/2017_000695.

Conflicts of Interests: The Authors declare that there are no competing interests.

This article is licensed under a Commons Attribution Non-Commercial 4.0 License

\section{ABSTRACT}

A healthy 44-year-old man, with a typical presentation of exertional heatstroke, presented with an increase in serum procalcitonin (PCT) levels. Suspecting a coexisting infection, the emergency physician started empirical antibiotic treatment and obtained samples for culture. The antibiotic treatment was stopped immediately after ward admission due to lack of signs of infection. The patient recovered completely after several days of rehydrating therapy and cultures remained negative for bacterial growth. PCT elevation is typically found with heatstroke, but this association is rarely found in daily practice due to the futility of PCT testing in this situation. Increased serum PCT levels in the context of heatstroke must not lead to an unsupported alternative diagnosis and useless investigations, tests and treatment.

\section{LEARNING POINTS}

- Elevated serum procalcitonin (PCT) levels are typical in heatstroke, although the association of PCT with heatstroke is rarely noted in clinical practice.

- A typical heatstroke scenario is enough to explain the increase in procalcitonin levels, and must not lead to inappropriate alternative diagnoses, investigations and treatments.

\section{KEYWORDS}

Heat stroke; procalcitonin; systemic inflammatory response syndrome

\section{CASE REPORT}

A previously healthy 44-year-old man took part in a $54 \mathrm{~km}$ run, with a difference in height of $3,900 \mathrm{~m}$, on a hot late spring day (over $30^{\circ} \mathrm{C}$ ) in southern Switzerland. He was found at $50 \mathrm{~km}$ confused and unable to stand unsupported. Medical personnel noted a Glasgow Coma Scale of 14/15 without focal neurological signs, moderate tachycardia (130 bpm), stable blood pressure and respiratory parameters, hot skin, and blood glucose $131 \mathrm{~g} / \mathrm{dl}$. The patient's body temperature was not recorded.

He was transported to the emergency department, while receiving $700 \mathrm{ml}$ of crystalloid infusion during the 45 -minute journey.

On arrival, the patient was still confused (GCS 14/15), but normocardic. His tympanic temperature was $38^{\circ} \mathrm{C}$ and the remaining clinical examination was unremarkable. Laboratory tests revealed a severe increase in creatinine (3.08 mg/dI) and creatinine kinase (CK; $3093 \mathrm{IU} / \mathrm{I})$. Procalcitonin, which was accidentally requested, was markedly elevated ( $36.7 \mu \mathrm{g} / \mathrm{l})$, although the leucocyte count and C-reactive protein (CRP) were in the normal range. Chest x-ray and urine examination did not show any signs of inflammation. The emergency physician recognized the heatstroke with acute renal failure and rhabdomyolysis, continued the treatment with crystalloid infusion and hospitalized the patient. However, because of the suspicion of a concurrent infection responsible for the PCT elevation, the physician started empirical antibiotic therapy with piperacillin/tazobactam and obtained blood and urine samples for culture. However, the ward physician, on admission 
of the patient, stopped the antibiotic therapy, due to the lack of evidence of infection, but continued the rehydrating therapy. The patient gradually returned to a normal cognitive status, and creatinine and CK (which peaked at 43,440 IU/I) slowly improved. PCR mildly increased on the second day $(21 \mathrm{mg} / \mathrm{l})$, but then returned to within the normal range. Blood and urine cultures did not show any bacterial growth. PCT was not measured again.

\section{DISCUSSION}

Heatstroke is characterized by hyperpyrexia and neurological symptoms such as delirium, convulsions or coma. It is caused by exposure to high temperatures (classic heatstroke) or by strenuous exercise (exertional heatstroke).

Physiologically, the body responds to heat with thermoregulatory adaptations mediated by the hypothalamus (e.g. cutaneous vasodilatation, sweating, reduction in visceral perfusion and increased minute ventilation) and the acute phase response, mediated by cytokines, that promotes protection against injury and repair. Heatstroke is the result of thermoregulatory failure and an exaggerated acute phase reaction $^{[1]}$.

Procalcitonin is a biological marker of systemic inflammation, in particular of bacterial origin, and is useful in clinical practice especially for the diagnosis and prognosis of bacterial infection.

Three published studies ${ }^{[2,3,4]}$ have shown that heatstroke is associated with elevation of serum PCT, but the prognostic significance is not clear. The physiopathological link between heatstroke and procalcitonin could be mediated by the exaggerated systemic inflammatory reaction that characterizes heatstroke.

Serum PCT measurement is considered futile and, consequently, seldom performed unless inflammation of bacterial origin is suspected. Therefore, although the PCT-heatstroke association is typical, PCT elevation is an uncommon finding in heatstroke, leading to incorrect and inappropriate alternative diagnoses, diagnostic tests and treatments.

In conclusion, in typical heatstroke, like exertional heatstroke, an increase in PCT must not lead to an unsupported suspicion of bacterial infection and does not justify microbiological investigations and empirical antibiotic treatment.

\section{REFERENCES}

1. Bouchama A, Knochel JP. Heat stroke. N Eng J Med 2002;346:1978-1988.

2. Hausfater P, Hurtado M, Pease S, Juillien G, Lvovschi VE, Salehabadi S, et al. Is procalcitonin a marker of critical illness in heatstroke? Intensive Care Med 2008;34:1377-1383.

3. Nylén ES, Al Arifi A, Becker KL, Snider RH Jr, Alzeer A. Effect of classic heatstroke on serum procalcitonin. Crit Care Med 1997;25:1362-1365.

4. Tong HS, Liu YS, Wen Q, Tang YQ, Yuan FF, Su L. Serum procalcitonin predicting mortality in exertional heatstroke. Emerg Med J 2012;29:113-117. 